\title{
Ketamine-induced Changes in Blood Pressure and Heart Rate in Pre-hospital Intubated Patients
}

\author{
Milan Kirit Sheth ${ }^{1 *}$, Asher Brand ${ }^{2}$, Julia Halterman ${ }^{1}$ \\ ${ }^{1}$ Department of Biology and Biomedicine, Eastern Mennonite University, \\ 1200 Park Road Harrisonburg, VA 22802, USA \\ ${ }^{2}$ PHI Air Medical, $2800 \mathrm{~N} \mathrm{44}{ }^{\text {th }}$ Suite 800 Phoenix, Arizona 85008, USA
}

dai: https://doi.org/10.21467/ajgr.3.1.20-33

* Corresponding Authar email: milan.sheth@emu.edu

Article Histary

Received: 27 September 2017

Revised: 02 October 2017

Accepted: 03 October 2017

Published: 04 October 2017

Student(s)

- $\quad$ Milan Kirit Sheth

Academic Year: Spring 2017

Course Level: Master Degree

Course Name: M. A.

(Master of Arts in Biomedicine) Course year: $2^{\text {nd }}$ Year

Mentar(s)

- Asher Brand

- Julia Halterman

\begin{abstract}
When a patient experiences trauma, pre-hospital rapid sequence intubation (RSI) is often the course of action. While RSI is associated with favorable neurological outcomes among traumatic brain injury patients, it also carries the risk of increased hypotension and bradycardia. Ketamine is a fast-acting anesthetic agent used in RSI, attractive due to its ability to raise heart rate (HR) and blood pressure (BP) and thus potentially lessening the severity of RSIrelated hypotension and bradycardia. However, research in support of its use is inconclusive, with different studies reporting increases or decreases in BP and HR after ketamine administration. To study the effect of ketamine on HR and BP during RSI, we analyzed the data obtained from 1,516 patients who received trauma and nontrauma medical care including RSI from first responders and emergency personnel using two different statistical methods. We determined if there was any association between the vital signs prior to administration of ketamine and outcomes such as bradycardia and/or hypotension post-ketamine administration in patients undergoing RSI. We found that the vital signs prior to administration of ketamine (i.e. BP and HR) predict the development of bradycardia and/or hypotension in patients undergoing RSI and the relationship between baseline vital signs and the development of bradycardia and/or hypotension is moderated by the dose of ketamine. Results obtained from this study may help responders and emergency care personnel to identify the patients that are likely to benefit from ketamine as an anesthetic agent.
\end{abstract}

Keywords: Ketamine, Blood Pressure, Heart Rate, Hypotension, Bradycardia, Rapid Sequence Intubation 


\section{Introduction}

Acute trauma patients often require pre-hospital advanced trauma life support, including intubation, during which a plastic tube is inserted into the trachea to keep the airway open or to serve as a channel for administering certain drugs. In most emergency cases, the best technique of choice is rapid sequence intubation (RSI). RSI involves administration of a fast-acting sedative (i.e. induction), along with a rapid neuromuscular blocker to facilitate endotracheal tube insertion [1]. Pre-hospital RSI is associated with a favorable neurological outcome over in-hospital intubation among traumatic brain injury patients [2]. RSI is a life-saving emergent procedure, yet it is not devoid of complications [3]. The complications that commonly occur with intubation and may be associated with a number of negative outcomes include: increased morbidity and mortality [4], oxygen desaturation [1], failed intubation, hypoxia, hypotension, and bradycardia [5]. A significant safety concern regarding sedative use in emergent conditions is the risk of hypotension and cardiovascular collapse especially in the critically ill such as trauma patients or those with pre-existing comorbid conditions. Furthermore, RSI may exacerbate hypotension in trauma patients taking benzodiazepines [6]. Despite these negative effects, the use of anesthesia remains necessary during intubation as it improves intubating conditions and minimizes procedure-associated adverse neuroendocrine responses that lead to hypertension and tachycardia and elevated intracranial pressure [7]. In order to optimize the performance of RSI and reduce complications, one must select the best sedative agent and dose most appropriate for the clinical scenario. Some of the commercially available sedatives that are used during intubation are etomidate, benzodiazepines, propofol, and barbiturates. Among the complications of RSI, hypotension (low BP) and bradycardia (low HR) are the key concerns for trauma surgeons. In head trauma patients, mortality rises from $1 \%$ in patients with hypoxia alone to $7 \%$ in patients with hypoxia and hypotension. Furthermore, more than one-half of head trauma patients experience bradycardia associated with RSI [8] . These statistics underscore the need to devise strategies to prevent or minimize these conditions among trauma patients who require intubation. This study aims to shed light on the cardiovascular effects of ketamine, a commonly used anesthetic in trauma patients, with particular focus on these two important outcomes.

Recently, ketamine has been gaining interest as a sedative agent in RSI. Ketamine effectively induces anesthesia, sedation, and amnesia without compromising airway protection or spontaneous respiratory efforts. It also exhibits a favorable hemodynamic profile making it an ideal agent for RSI in trauma patients [9]. Current literature reporting on the use of ketamine to facilitate ETI in the EMS setting remains scarce. Moreover, only a handful reported changes in hemodynamic measures due to ketamine, and those were inconclusive, with some studies reporting increases and others decreases in BP and HR. It is also unclear if varying baseline hemodynamic measurements alters the ketamine response. Ketamine was discovered in 1962 and following FDA approval was given to American soldiers during the Vietnam war. Ever since, it has been used as a widespread anesthetic and has been placed in the 'core' list in the WHO's essential drugs list [10]. Ketamine is a dissociative anesthetic that is administered intravenously and is available in multiple strengths, including $10 \mathrm{mg} / \mathrm{ml}, 50 \mathrm{mg} / \mathrm{ml}$, and $100 \mathrm{mg} / \mathrm{ml}$. Ketamine works within 5 minutes, and a dose ranging from $1-4 \mathrm{mg} / \mathrm{kg}$ is enough to induce a dissociative state [11]. Unlike other sedatives that depress the reticular activating system, ketamine affects the limbic system preventing sensory stimuli from traveling to the cerebral cortex. As a result, ketamine produces an anesthetic effect without impairing breathing. It also stimulates the brain stem leading to catecholamine release and inhibition of norepinephrine reuptake, ultimately causing an increase in heart rate (HR) and blood pressure (BP) [12]. Thus, if a patient demonstrates bradycardia and hypotension as a result of intubation, ketamine may potentially raise the HR and BP closer to normal levels [13] . Ketamine is also unique in that it provides analgesic effects along with its amnestic and sedative effects. These distinctive properties have made ketamine a compelling option for anesthesia induction in both pre-hospital and in-hospital settings. In this study, we examined the effect of ketamine on HR and BP and identified potential predictors of the occurrence of three important postketamine clinical outcomes: bradycardia, hypotension and hypertension. 
Sheth et al., Adv.J. Grad. Res.; Vol. 3 Issue 1, pp: 20-33, January 2018

\subsection{Effects of Ketamine on Vital Signs in the In-hospital Setting}

A number of studies have investigated the impact of ketamine on vital signs (VS) during in-hospital care. Ayatollahi et al. [14] compared ketamine, lidocaine, and ephedrine to placebo with regards to their effects on $\mathrm{BP}$ and HR in 140 surgical patients undergoing anesthesia using propofol. The researchers found that, compared to placebo, ketamine was associated with slightly lower systolic BP (SBP) but had no impact on HR [14] . Rajan et al. [15] randomized 40 patients undergoing caesarean section to receive either ketamine or normal saline (NS) prior to induction of anesthesia and intubation. VS of both groups were compared and it was found that ketamine was associated with significantly lower SBP and HR than placebo. HR and SBP were comparable between the ketamine and saline groups prior to intubation, but after intubation these variables were higher in the control group. Furthermore, HR and SBP were lower than the baseline readings in the ketamine group [15] . This study supports the BP lowering effect of ketamine reported by Ayatollahi et al. [14]. Other studies have reported conflicting results to Ayatollahi et al. [14] and Rajan et al. [15] with respect to ketamine's effect on BP and HR. Ozkan et al. [16] compared the effects of ketamine to placebo in 60 patients undergoing transurethral resection for benign prostatic hyperplasia. Patients who received ketamine demonstrated significantly higher mean arterial pressure (MAP), SBP, and HR than the placebo group [16]. Generally, patients receiving spinal anesthesia experience a drop in VS. In this study, the ketamine group experienced a less severe drop than the placebo group [16] . Similar findings were reported in a study of 54 children undergoing adenotonsillectomy who received either ketamine or alfentanil in adjunct to propofol and rocuronium for induction of anesthesia [17]. The researchers found that the ketamine group demonstrated an overall increase in BP and HR. The MAP initially dropped after intubation in the ketamine group but it rebounded within one minute to levels above baseline. Barois et al. [18] studied the effects of ketamine use prior to endotracheal intubation (ETI) in newborns. Ketamine was administered to 37 out of 59 preterm infants undergoing intubation. Newborns given ketamine were found to have significant increases in HR over the controls (150.7 versus $112.6 \mathrm{bpm}$ ) although BP was slightly lower in the ketamine group (30.0 versus $32.9 \mathrm{mmHg}$ ). Therefore, the authors suggested that ketamine given prior to intubation is effective in preventing bradycardia in pre-term infants [18] .

The effect of ketamine on HR and BP in trauma patients is somewhat inconclusive and controversial between studies. Previous studies regarding ketamine use during intubation in an in-hospital setting have reported variable results with respect to its effect on BP and HR. For example, Ayatollah et al. [14] and Rajan et al. [15] reported decreased BP and HR [15], [19] while Ozcan et al. [16] and Kim et al. [17], reported an increase in both of these parameters after ketamine administration. Barois et al. [18] reported decreased BP but an increase in HR. The differences in results reported by various studies could be due in part to differences in study populations (age, sex, co-conditions, etc.), indications of the anesthetic, route of administration, use of additional drugs, and perhaps dosing. In the study conducted by Ayatollah et al. [14] patients received a combination of three drugs, which could have potentially modified the ketamine response. Also, patient characteristics varied widely between the studies, notably in sex and age, for example Rajan et al. [15] studied pregnant women, Ozcan et al. [16] studied older men with BPH, Kim et al. [17] studied children and Barios et al. [18] studied neonates. Also, the route of administration differed between the studies. For example, Ozcan et al. [16] delivered spinal anesthesia while others gave injections.

\subsection{Effects of Ketamine on Vital Signs in the Pre-hospital Setting}

To date, very few studies have addressed the use of ketamine in the pre-hospital setting. Losvik et al. [20] compared the effects of ketamine, pentazocine, and placebo on VS in adult trauma patients prior to hospitalization. Compared with pentazocine and placebo, patients who received ketamine demonstrated an increase in BP [20] . Likewise, Tran et al. [21] compared the hemodynamic profiles of ketamine and morphine given for analgesia in 312 trauma patients prior to hospitalization. On average, patients who received ketamine had higher increases in BP from baseline than those who received morphine $(9.3 \mathrm{mmHg}$ vs. $8 \mathrm{mmHg}$ ). The authors concluded that ketamine provides a comparable analgesic effect to morphine, yet with a lower risk of airway complications and hypotension [21]. A review of 115 trauma patients who 
underwent pre-hospital intubation found that laryngoscopy and intubation caused significant increases in HR and MAP or SBP and that prior administration of $100 \mathrm{mg}$ of ketamine moderately decreased the SBP, and slightly decreased the MAP with a minimal to no effect on HR. However, none of these differences were statistically significant when compared to controls [7]. A similar discrepancy on the effects of ketamine was seen between studies pertaining to the use of ketamine for trauma patients in the pre-hospital setting. Both Lovsik et al. [20] and Tran et al. [21] reported increased BP. while Perkins et al. [7] reported decreased BP. The results of Perkins et al. [7] were not statistically significant and might have been underpowered. Nonetheless, there is a clear variation in the literature regarding VS changes associated with ketamine administration in trauma patients in the pre-hospital setting.

\subsection{Rationale and Hypothesis}

The discrepancies that exist in the literature surrounding the use of ketamine in RSI justify additional investigation. Researchers do not yet agree on how ketamine affects BP and HR. However, this information is of vital importance to first responders, surgeons, and emergency department personnel. If RSI increases mortality and the incidence of negative effects such as hypoxia, hypotension, and bradycardia, then surgeons must be able to anticipate which patients are at risk of developing such effects.

The goal of this study was to examine whether VS taken prior to ketamine administration for RSI can predict subsequent occurrence of hypotension and/or bradycardia in both trauma and medical patients in the pre-hospital setting. The research literature is unclear as to whether varying baseline hemodynamic measurements effect how patients respond to ketamine. When RSI leads to bradycardia and hypotension in a trauma patient and the use of ketamine raises both of these parameters, the use of ketamine becomes useful in the field and may benefit this particular subset of patients by bringing up their HR and BP. However, while this is the case in some studies, in others, ketamine seemed to have the opposite effect and caused both BP and HR to decrease. As a result, it may be difficult for first responders, ER personnel and trauma surgeons to determine who will benefit from ketamine's hypertensive and tachycardic effects versus those who will develop hypotension and/or bradycardia. Therefore, we hypothesized that baseline hemodynamic measures (VS) may predict how patients respond to ketamine administration in terms of changes in their BP and HR. To further clarify the determinants of the ketamine response in patients undergoing RSI we also looked at the effects of different doses on the hemodynamic response. This will enable healthcare professionals to improve upon current mortality and morbidity rates associated with RSI. Furthermore, the results of our study may also potentially aid physicians in identifying patients in whom ketamine is likely to induce a favorable effect from those who are likely at risk of developing hemodynamic instability and subsequent complications. Our results also outline the importance of categorizing and carefully looking at the characteristics of each patient before deciding the way to go in terms of airways protection. While follow-up studies are required before this idea goes into practice, our study provides a better understanding of the factors that predict unfavorable outcomes following ketamine induction.

\section{Methods}

\subsection{Definitions}

For the purpose of this study the following definitions were used: RSI refers to the administration of a sedative (in this case ketamine) to induce general anesthesia prior to ETI; Bradycardia is a $\mathrm{HR}<60$; Hypotension is a MAP $\leq 70$; Normotension is a SBP $<130$ and $>90$ and a diastolic BP (DBP) $<85$ and $>$ 60; Hypertension is a MAP of $\geq 105$; and the Shock Index (SI) is defined as the HR/ SBP (normal SI= 0.5 - 0.7) and it indicates the level of occult shock. The MAP was derived from the SBP and DBP using the following formula: $M A P=1 / 3(S B P-D B P)+D B P$. Baseline HR, SBP and DBP measurements were recorded prior to RSI. 
Sheth et al., Adv.J. Grad. Res.; Vol. 3 Issue 1, pp: 20-33, January 2018

\subsection{Study Design and Data Collection}

Table 1: Descriptive Characteristics of Study Sample

The study is a retrospective analysis of data obtained from PHI Air Medical (PHI). PHI is a helicopter emergency medical service (HEMS) that safely transports more than 30,000 patients yearly across the United States. Both trauma and medical patients who underwent RSI between 2013 and 2016 and who received ketamine as their main induction drug were included in the study. Patients were categorized into four groups according to their received ketamine doses: < 100 $\mathrm{mg} / \mathrm{mL}, \quad 101-150 \mathrm{mg} / \mathrm{mL}, \quad 151-200$ $\mathrm{mg} / \mathrm{mL}$, and $>200 \mathrm{mg} / \mathrm{mL}$. The study was non-interventional in the sense that each patient received medical care as per protocol and patient data was collected and entered into medical records at the time of HEMS transport as per usual practice of PHI. Data regarding patient demographics, patient category (medical vs. trauma), drug doses and hemodynamic measures were obtained. Patients with no pulse or an un-recordable BP at baseline or after ketamine administration were excluded.

\begin{tabular}{|c|c|c|c|}
\hline Characteristics & Mean \pm SD & $\mathbf{n}$ & $\%$ \\
\hline \multicolumn{4}{|l|}{ Socio demographics } \\
\hline Age & $50.5 \pm 20.3$ & & \\
\hline \multicolumn{4}{|l|}{ Patient type } \\
\hline Medical & & 730 & 48.2 \\
\hline Trauma & & 786 & 51.8 \\
\hline \multicolumn{4}{|l|}{ Pre-ketamine vital signs \& dose } \\
\hline Systolic BP & $126.3 \pm 33.6$ & & \\
\hline Diastolic BP & $76.2 \pm 20.4$ & & \\
\hline Heart Rate (HR) & $100.8 \pm 25.7$ & & \\
\hline Mean arterial pressure (MAP) & $92.9 \pm 23.9$ & & \\
\hline Shock index & $1.35 \pm .57$ & & \\
\hline \multicolumn{4}{|l|}{ Ketamine dose } \\
\hline 100 or lower & & 266 & 17.5 \\
\hline 101 to 150 & & 489 & 32.3 \\
\hline 151 to 200 & & 629 & 41.5 \\
\hline above 200 & & 132 & 8.7 \\
\hline \multicolumn{4}{|l|}{ Post-ketamine vital signs } \\
\hline Systolic BP & $122.2 \pm 33.5$ & & \\
\hline Diastolic BP & $75.6 \pm 21.2$ & & \\
\hline Heart Rate & $101.6 \pm 24.6$ & & \\
\hline Mean arterial pressure (MAP) & $91.1 \pm 24.6$ & & \\
\hline Shock index & $1.28 \pm .53$ & & \\
\hline \multicolumn{4}{|l|}{ Post-ketamine clinical outcome } \\
\hline Bradycardia & & 141 & 9.3 \\
\hline Hypotension & & 548 & 36.1 \\
\hline Hypertension & & 781 & 51.5 \\
\hline
\end{tabular}
There were no exclusion criteria based on patient demographics, diagnosis and associated medical conditions, and concurrent medications or interventions.

A total of 1,516 patients were included in the study. Of these, 730 were medical patients and 786 were trauma patients. The mean age of patients was $50.5 \pm 20.3$ years (range, 7-94 yrs). The average values for pre-ketamine vital signs (SBP, DBP, HR, MAP and SI) as well as the frequencies for post-ketamine hemodynamic outcomes (bradycardia, hypotension, and hypertension) are shown in Table 1. The most frequently administered ketamine dose was $150-200 \mathrm{mg} / \mathrm{mL}(\mathrm{n}=629,41.5 \%$ ), followed by $101-150 \mathrm{mg} / \mathrm{mL}$ $(\mathrm{n}=489,32.3 \%)$, then $\leq 100 \mathrm{mg} / \mathrm{ml}(\mathrm{n}=266,17.5 \%)$ and lastly $>200 \mathrm{mg} / \mathrm{ml}(\mathrm{n}=132,8.7 \%)$ (Table 1). The Shapiro Wilk test for normality showed post-ketamine pulse (HR) to be normally distributed $(\mathrm{p}=0.3)$ while all other vital signs were not normally distributed $(\mathrm{p}<0.01)$. But, given the large sample size $(>1000)$, we decided to report parametric tests for bivariate analysis despite our data being non-normal. Additionally, we performed the non-parametric equivalent for all comparisons.

\subsection{Outcome Measures}

Three primary outcome variables (i.e., response variables) were tested. These variables reflect changes in hemodynamic measurements following administration of ketamine, namely, bradycardia, hypotension, and hypertension. Independent (i.e., predictive) variables tested were baseline (pre-ketamine) hemodynamic measures (SBP, DBP, HR, MAP and SI), patient age and patient category (medical vs. trauma). Body weight was missing in a number of study participants and was therefore excluded from the analysis. 


\subsection{Statistical Analyses}

Data was cleaned and organized to only represent pre-ketamine VS and VS after the first dose of ketamine and before the second shot of ketamine was administered (if any second dose was needed). Normality was assessed using the Kolmogorov-Smirnov test and Shapiro Wilk test. Where appropriate, the chi-square $(\chi 2)$ or Fisher's exact test were used to compare categorical data (i.e. patient category versus primary outcome). The independent samples t-test or its non-parametric equivalent, the Mann-Whitney U-test, were used to compare continuous variables across two groups. Paired data were analyzed using a paired t-test or the nonparametric Wilcoxon Signed-Rank test when the normality assumption was not met. A hierarchical logistic regression model was developed to determine the predictors of bradycardia, hypotension and hypertension after ketamine administration. The covariates, age and patient category, were included in the first block, followed by the predictors (pre-ketamine vital signs) in the second block. Dose, the moderator, was included in the third block. Finally, the interaction terms between dose and the pre-ketamine vital signs were included in a fourth block. Results of the logistic regression model were reported as adjusted odds ratio (OR) with corresponding 95\% CIs. Statistical significance was established at a 5\% level (i.e., p-value of $<0.05$ ) for all tests. Some limitations of our study are: The inability to control data collection due to the retrospective design of our study; Not assessing for tachycardia, gender, body weight, co-morbidities, and adjunctive drugs including I.V. fluids and other sedatives; Assessing one point in time without indexing; Not considering other complications of intubation that can potentially alter patient's hemodynamics such as intubation difficulties and hypoxia; Lastly, lack of a control group (perhaps "etomidate" the gold standard) which reduces it applications.

An important strength of our study is the big sample size (1,516 patients) that increases the possibility of generalized conclusions. Another strength of our study is that we looked at how baseline variations in VS may predict post-ketamine clinical outcomes, which most studies have not looked at. Testing composite hemodynamic indices such as MAP and the SI has added value to the applications of our study, for instance MAP is a better indicator of cerebral perfusion pressures and therefore including it in the analysis adds great value in guiding management in brain injury patients. Also, the SI helps us better stratify at-risk patients even when baseline and post-ketamine VS are normal. It is important to remember that the SI can be abnormal even when both the BP and HR are normal [22]. Further studies including these indices especially the SI would be needed to better determine their usefulness in trauma practice and in guiding anesthetic use.

\section{Results}

\subsection{Relationship between Pre-Ketamine Vital Signs, Demographic Variables and Post-Ketamine} Bradycardia

The development of bradycardia following ketamine administration was significantly associated with baseline (pre-ketamine) VS measures (Figure 1). Patients who experienced bradycardia had significantly lower pre-ketamine SBP (Mean= $111.6 \mathrm{mmHg}$ vs. $127.8 \mathrm{mmHg}, \mathrm{p}<.001)$, DBP (Mean= $66.74 \mathrm{mmHg}$ vs. $77.2 \mathrm{mmHg}$; $\mathrm{p}<.001)$, MAP $(81.0 \mathrm{mmHg}$ vs. 94.1 $\mathrm{mmHg} ; \mathrm{p}<.001)$, and HR $(82.7 \mathrm{bpm}$ vs. $102.6 \mathrm{bpm} ; \mathrm{p}<.001)$ compared to patients who did not experience bradycardia. Patients who experienced post-ketamine bradycardia also had significantly higher pre-ketamine SI

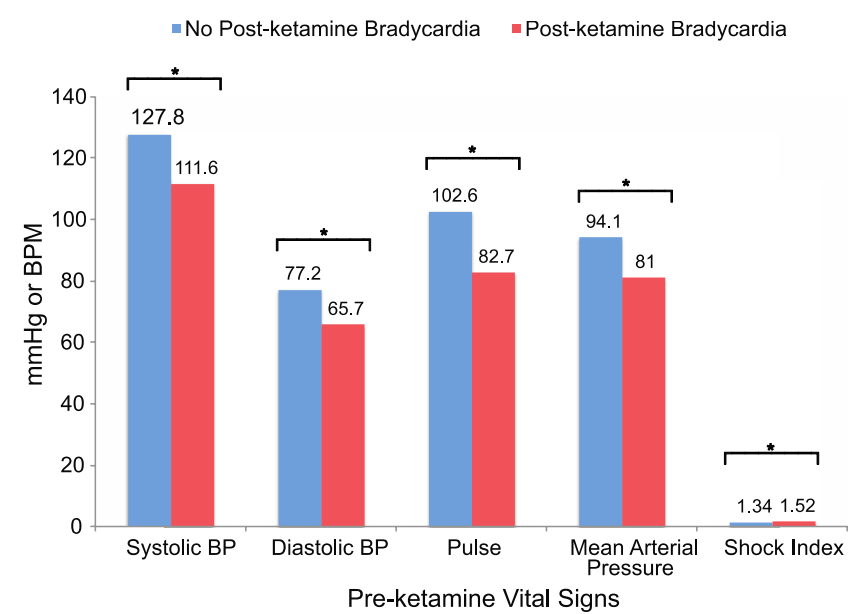

Figure 1: The Relationship between Pre-Ketamine Vital Signs and Post-Ketamine Bradycardia. The asterisk represents statistically significant comparisons $(p<.001$ for all). 
Sheth et al., Adv.J. Grad. Res.; Vol. 3 Issue 1, pp: 20-33, January 2018

values compared to those who did not experience bradycardia (1.52 mmHg vs. $1.34 \mathrm{mmHg}$; $\mathrm{p}=.002)$. On average, patients who experienced bradycardia were 13 years older than those who did not (62.2 years vs. 49. 3 years; $\mathrm{p}<.001$ ) (Figure 2). A significantly higher proportion of medical patients developed bradycardia after ketamine when compared to trauma patients $(12.2 \%(\mathrm{n}=89)$ vs. $6.6 \% \%(\mathrm{n}=52 / 786)$, Chi-square $=13.9, \mathrm{p}<.001)$ (Figure 3). P-values obtained from the nonparametric test (Mann Whitney-U Test for continuous data, and Fischer exact test for categorical data) were consistent with the results of the parametric tests.

The logistic regression model for the prediction of post-ketamine bradycardia showed both MAP and SI to be significant predictors for development of bradycardia post-ketamine administration (Table 2). MAP was a negative predictor of bradycardia as indicated by an OR $<1$ [OR $0.962,95 \%$ CI $(0.947$, 0.976); $\mathrm{p}<.001]$. SI, on the other hand, was a positive predictor of bradycardia as indicated by an $\mathrm{OR}>1$. In particular, according to the model, a unit increase in the SI increases the odds of experiencing bradycardia by more than two-folds [OR 2.61, 95\% CI $(1.35,5.06) ; \mathrm{p}=.004)]$. Age was found to be positively associated with bradycardia [OR 1.02, 95\% CI (1.01, 1.04), $\mathrm{p}<.001)]$. Dose (the moderator) was also significantly associated with bradycardia [OR 1.77, 95\% CI $(1.08,2.89), \mathrm{p}=.023)]$.

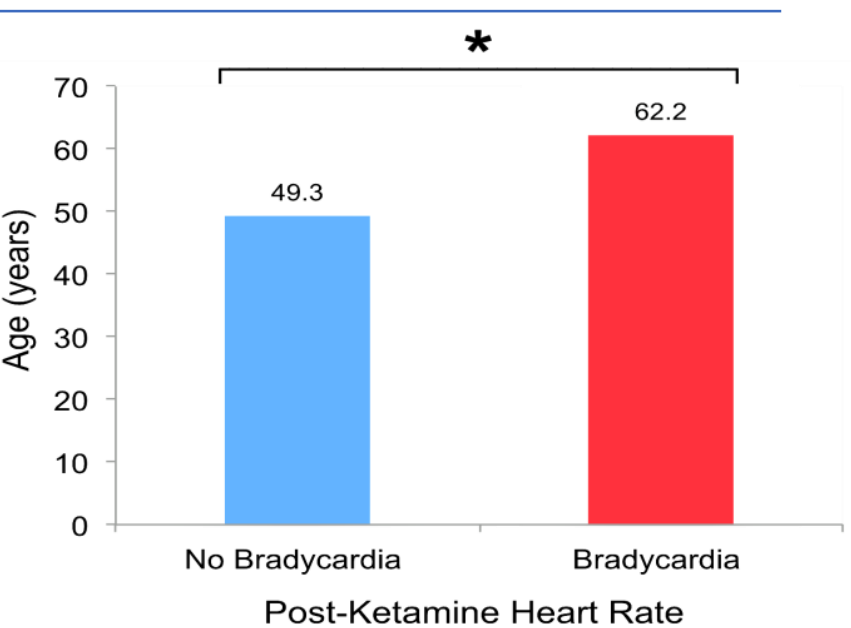

Figure 2: The Relationship between Age and Post-Ketamine Bradycardia. Patients who experienced bradycardia were significantly older than patients who did not $(p<.001)$.

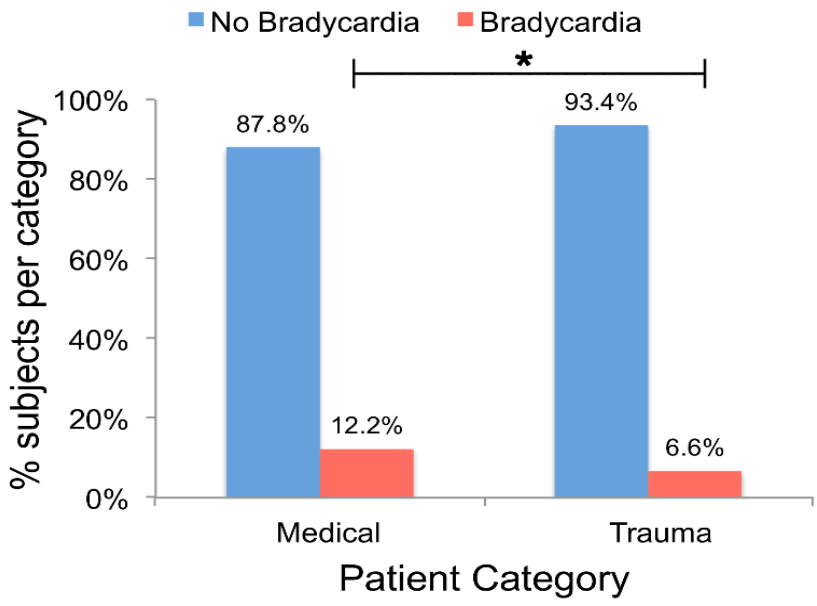

Figure 3: The Relationship between Patient Category and PostKetamine Bradycardia. Medical patients were significantly more likely to experience bradycardia after ketamine administration than trauma patients $(p<.001)$.

Table 2: Logistic Regression for pre-Ketamine Vital Signs Predicting Bradycardia, controlling for Age and Patient Category.

\begin{tabular}{|l|l|l|l|l|}
\hline Variables $^{a b}$ & OR & $95 \%$ CI & Wald & p-value \\
\hline Age & 1.02 & {$[1.01,1.04]$} & 17.4 & .000 \\
\hline Trauma & 1.04 & {$[.683,1.58]$} & .032 & .859 \\
\hline Pre MAP & .962 & {$[.947, .976]$} & 25.6 & .000 \\
\hline Pre pulse & .987 & {$[.973,1.00]$} & 3.06 & .080 \\
\hline Pre shock index & 2.61 & {$[1.35,5.06]$} & 8.14 & .004 \\
\hline Dose & 1.77 & {$[1.08,2.89]$} & 5.14 & .023 \\
\hline Pre MAP*dose & 1.02 & {$[.987,1.05]$} & 1.24 & .266 \\
\hline Pre pulse*dose & .984 & {$[.956,1.01]$} & 1.32 & .251 \\
\hline Pre shock*dose & .236 & 4.57 & .033 \\
\hline
\end{tabular}

Finally, when including the interaction terms for the moderator (dose) in block 4, there was a significant gain at a $10 \%$ level but not at a $5 \%$ level (Chi-square $=7.35, \mathrm{p}=.064)$. However, the Wald test for the interaction terms showed that the interaction between dose and the $\mathrm{SI}$ is significant $(\mathrm{OR}=0.236,95 \% \mathrm{CI}$ 
$(.063, .887) ; \mathrm{p}=.033)$. The moderation effect is represented graphically in Figure 4. In particular, the relationship between SI and the $\log$ of odds of bradycardia is positive and stronger for low doses of ketamine represented by the solid line. For high doses of ketamine, the relationship between SI and bradycardia becomes weaker as indicates the flatter discontinuous line.

\subsection{Relationship between Pre-Ketamine Vital Signs, Demographic Variables, and Hypotension}

There were significant differences in all baseline VS, except HR, between patients who experienced hypotension after ketamine administration and those who did not (all pvalues < .001). Patients who experienced hypotension had significantly lower baseline SBP, DBP, MAP and SI compared (Figure 5). Additionally, significant differences in age were observed between patients who experienced hypotension and those who did not (Figure 6). Those who experienced hypotension were on average, about 10 years older than those who didn't. These results were consistent with the non-parametric tests. Under the Mann-Whitney U, however, the differences in HR were also significant with the hypotensive group showing slightly larger values for baseline HR ( $\mathrm{p}=.029)$.

Medical patients were significantly more likely to experience hypotension after ketamine administration than trauma patients (Chisquare $=36.1, \mathrm{p}<.001)$. In particular, $43.8 \%$ $(\mathrm{n}=320)$ of medical patients experienced hypotension after ketamine administration whereas only $29 \%(n=228)$ of trauma patients experienced hypotension (Figure 7).

A logistic regression model for potential predictors of post-ketamine hypotension showed age [OR 1.03, 95\% CI $(1.02,1.03) ; \mathrm{p}$ $<.001)$ ] and ketamine dose [OR 1.3, 95\% CI $(1.01,1.7) ; \mathrm{p}=0.04]$ to be positive predictors of hypotension and MAP [OR 0.951, 95\% CI $(0.94,0.96) ; \mathrm{p}<.001)]$ a negative predictor of hypotension.

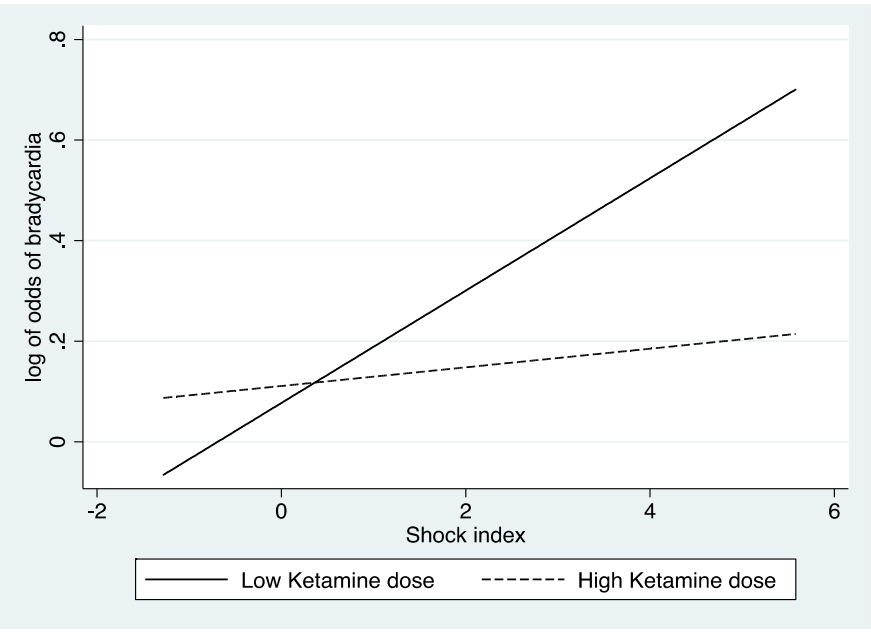

Figure 4: Moderation Effect of Dose on Shock Index as a Predictor of Bradycardia.

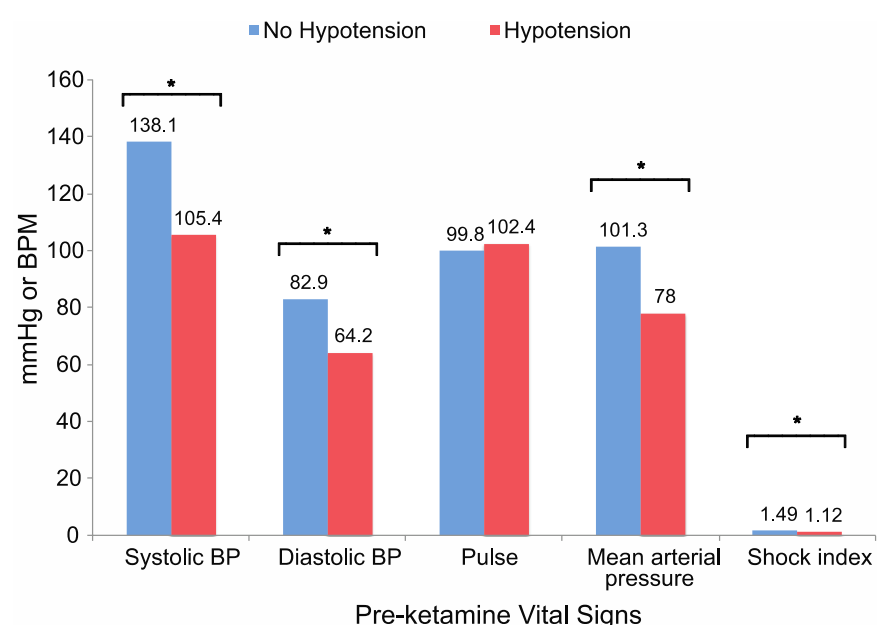

Figure 5: The Relationship between Pre-Ketamine Vital Signs and Post-Ketamine Hypotension. The asterisk represents statistically significant comparisons ( $p<.001$ for all).

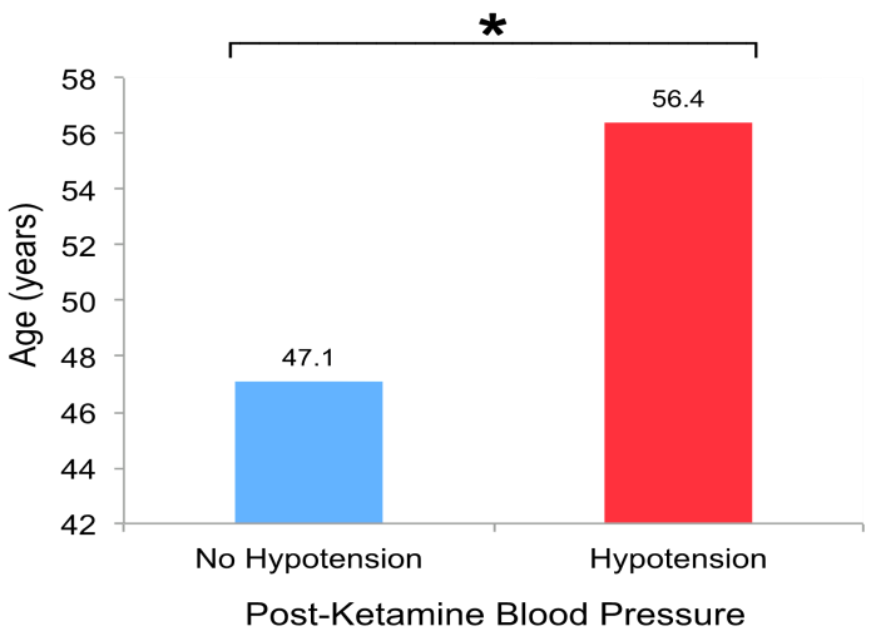

Figure 6: The Relationship between Age and Post-Ketamine Hypotension. Patients who experienced hypotension were significantly older than patients who did not $(p<.001)$. 
Sheth et al., Adv.J. Grad. Res.; Vol. 3 Issue 1, pp: 20-33, January 2018

\subsection{Relationship between Pre-Ketamine Vital Signs, Demographic Variables, and Hypertension}

There were significant differences in all baseline VS, except HR (pulse), between patients who experienced hypertension after ketamine administration and those who did not (all pvalues < .001). In particular, patients who experienced hypertension after ketamine administration had higher values of BP (SBP, DBP and MAP) and higher SIs before ketamine administration (Figure 8). Those who experienced hypertension were also on average four years younger (Figure 9). These findings were consistent with those obtained from the nonparametric tests.

Trauma patients were significantly more likely to experience hypertension after ketamine administration than medical patients (Chi-square $=46.18, \mathrm{p}<.001$ ). In particular, $59.9 \%(n=471)$ of trauma patients experienced hypertension after ketamine administration whereas only $42.5 \%(n=310)$ of medical patients did (Figure 10).

A logistic regression model for potential predictors of post-ketamine hypertension showed trauma patients to be 1.67 times more likely to develop hypertension than medical patients [OR 1.67, 95\% CI (1.31, 2.13); p < .001)]. It also showed pre-ketamine MAP to be a slight positive predictor of hypertension after ketamine administration [OR 1.03, 95\% CI $(1.02,1.04): \mathrm{p}<.001)]$. Higher ketamine dose $(>150 \mathrm{mg})$ was also a positive predictor of hypertension [OR 1.3, 95\% CI (1.04, 1.62); $\mathrm{p}=.02)]$.

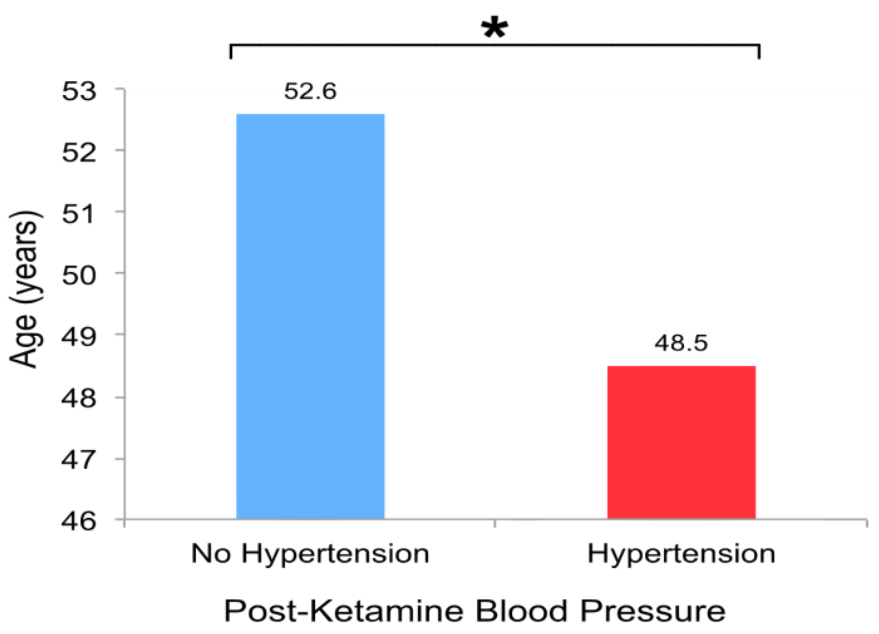

Figure 9: The Relationship between Age and Post-Ketamine Hypertension. Patients who experienced hypertension were significantly younger than patients who did not ( $p<.001)$.

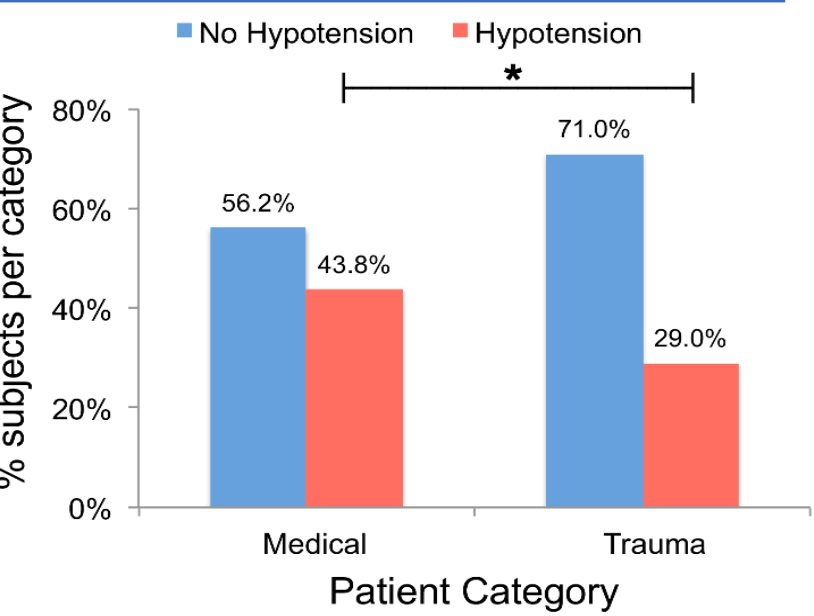

Figure 7: The Relationship between Patient Category and PostKetamine Hypotension. Medical patients were significantly more likely to experience hypotension after ketamine administration than trauma patients $(p<.001)$. -No Hypertension $\quad$ Hypertension

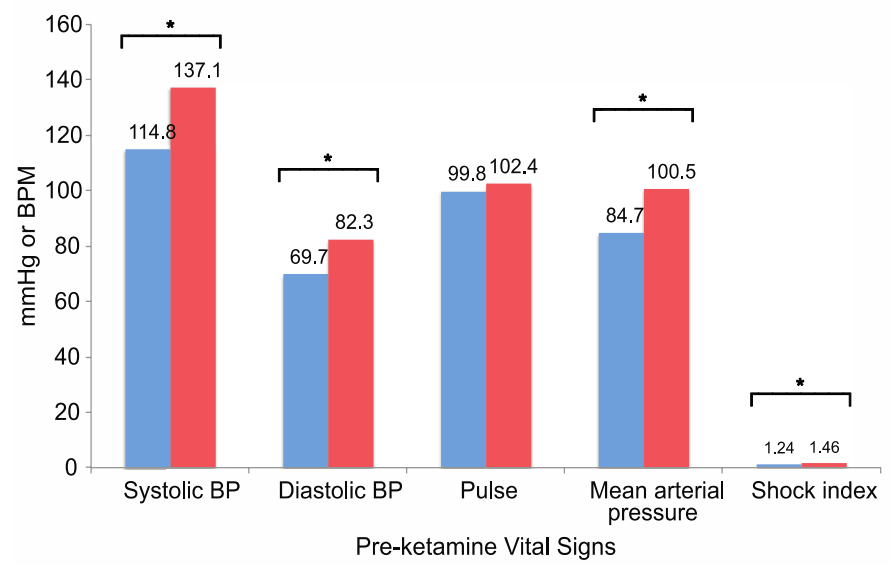

Figure 8: The Relationship between Pre-Ketamine Vital Signs and Post-Ketamine Hypertension. The asterisk represents statistically significant comparisons ( $p<.001$ for all).

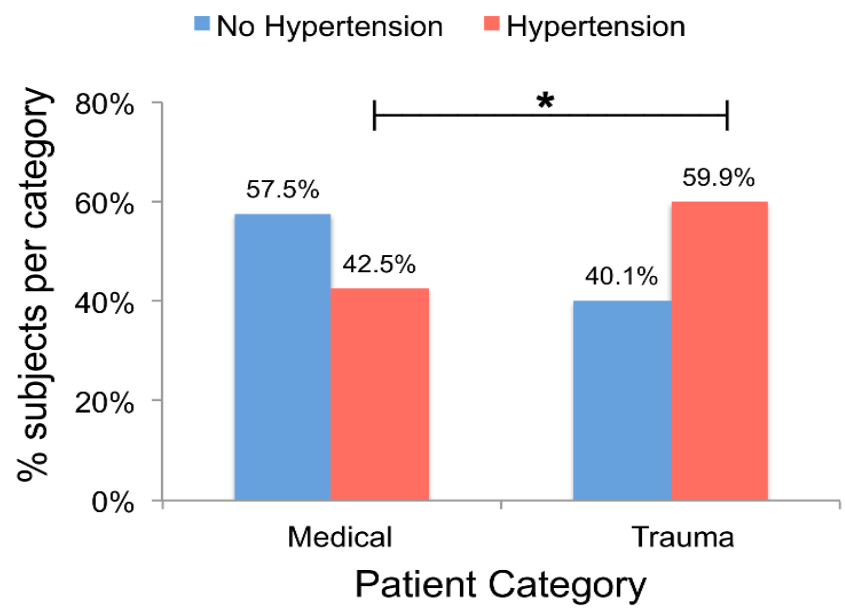

Figure 10: The Relationship between Patient Category and Post-Ketamine Hypertension. Trauma patients were significantly more likely to experience hypertension after ketamine administration than medical patients $(p<.001)$. 


\subsection{Comparing Vital Signs Before and After Ketamine}

There was a significant difference between pre-ketamine and post-ketamine SBP, MAP, and SI when paired hemodynamic measures of the same individual were compared. Following ketamine administration, on average, the SBP decreased by 4 units ( $p<.001$, paired-t-test), the MAP decreased by 1.75 units $(p<.001$, paired-t-test) and the SI decreased by 0.07 units $(\mathrm{p}<.001$, paired-t-test). These findings were consistent with those obtained from the equivalent non-parametric test, the Wilcoxon signed ranks test (Table 3).

Table 3: Paired T-test Comparing Vital Signs Before and After Ketamine

\begin{tabular}{|l|r|r|r|r|}
\hline Vital signs & \multicolumn{1}{|c|}{$\begin{array}{c}\text { Pre-Ketamine } \\
\text { mean } \pm \text { SD }\end{array}$} & $\begin{array}{c}\text { Post-Ketamine, } \\
\text { mean } \pm \text { SD }\end{array}$ & p-value $^{\mathbf{1}}$ & p-value $^{\mathbf{2}}$ \\
\hline Systolic BP & $126.3 \pm 33.6$ & $122.2 \pm 33.5$ & .000 & .000 \\
\hline Diastolic BP & $76.2 \pm 20.4$ & $75.6 \pm 21.2$ & .252 & .502 \\
\hline Pulse (HR) & $100.1 \pm 25.7$ & $101.6 \pm 24.6$ & .131 & .127 \\
\hline Mean arterial pressure (MAP) & $92.9 \pm 23.9$ & $91.1 \pm 24.6$ & .001 & .009 \\
\hline Shock index & $1.35 \pm .55$ & $1.28 \pm .53$ & .000 & .000 \\
\hline \multicolumn{2}{|r}{} \\
\hline
\end{tabular}

\subsection{Relationship between Ketamine Dose and Post-Ketamine Clinical Outcomes}

Table 4 shows the relationship between ketamine dose and post-ketamine clinical outcomes. There was a significant association between ketamine dose and the development of post-ketamine bradycardia (Chisquare $=19.3, \mathrm{p}<.001)$ and hypertension (Chi-square $=19.3, \mathrm{p}=.001)$. Patients who received higher doses of ketamine were more likely to experience bradycardia and hypertension. It was found that only $8 \%$ of patients who did not experience bradycardia received a ketamine dose $\geq 200 \mathrm{mg}$ while $16 \%$ of those who experienced bradycardia received a dose of ketamine $\geq 200 \mathrm{mg}$ (Figure 11). Patients who developed hypertension received moderately higher ketamine doses than patients who did not develop hypertension, with most doses being between 151-200 mg (Figure 11). The development of post-ketamine hypotension was not affected by differences in the ketamine dose (Chisquare $=19.3, \mathrm{p}=.116$ ).

\section{Bradycardia}

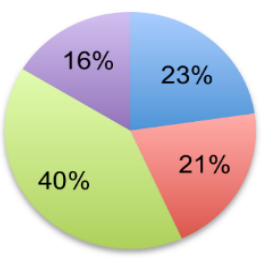

Hypotension

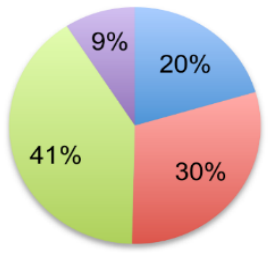

Hypertension

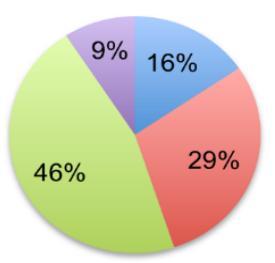

No Bradycardia

$$
\begin{aligned}
& =\leq 100 \mathrm{mg} \\
& =101-150 \mathrm{mg} \\
& =151-200 \mathrm{mg} \\
& =>200 \mathrm{mg}
\end{aligned}
$$

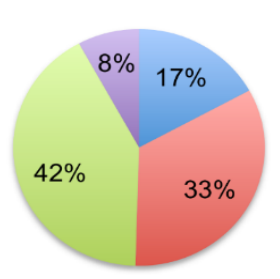

No Hypotension

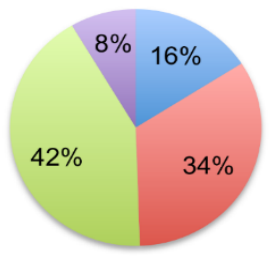

No Hypertension

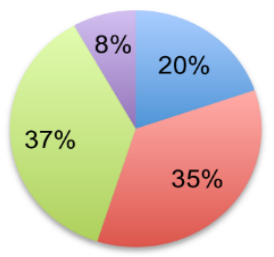

Figure 1: Differences in Ketamine Doses across Different Clinical Outcomes

Table 4: Relationship between Ketamine Dose and Study Outcomes

\begin{tabular}{|l|c|c|c|c|c|c|c|}
\hline & & \multicolumn{4}{|c|}{ Clinical Outcome } \\
\hline Ketamine dose & $\begin{array}{c}\text { Total Number } \\
\text { of Patients (n) }\end{array}$ & \multicolumn{2}{|c|}{$\begin{array}{c}\text { Bradycardia } \\
\text { n (row \%) }\end{array}$} & \multicolumn{2}{c|}{$\begin{array}{c}\text { Hypotension } \\
\text { n (row\%) }\end{array}$} & \multicolumn{2}{c|}{$\begin{array}{c}\text { Hypertension } \\
\text { n (row\%) }\end{array}$} \\
\hline & & Yes & No & Yes & No & Yes & No \\
\hline$\leq 100 \mathrm{mg}$ & 266 & $32(12)$ & $234(88)$ & $112(42.1)$ & $154(57.9)$ & $121(45.5)$ & $145(54.5)$ \\
\hline $101-150 \mathrm{mg}$ & 489 & $29(5.9)$ & $460(94.1)$ & $164(33.5)$ & $325(66.5)$ & $230(47)$ & $259(53)$ \\
\hline $151-200 \mathrm{mg}$ & 629 & $57(9)$ & $572(91)$ & $222(35.3)$ & $407(64.7)$ & $358(57)$ & $271(43)$ \\
\hline$>200 \mathrm{mg}$ & 132 & $23(17.4)$ & $109(82.6)$ & $50(37.9)$ & $82(62.1)$ & $72(54.5)$ & $60(45.5)$ \\
\hline
\end{tabular}


Sheth et al., Adv.J. Grad. Res.; Vol. 3 Issue 1, pp: 20-33, January 2018

\section{Discussion}

Out of a total of 1,516 patients, 141 (9.3\%) experienced bradycardia, 548 (36.1\%) experienced hypotension and $781(51.5 \%)$ experienced hypertension following ketamine administration (Table 1). On bivariate analysis, lower baseline (pre-ketamine) HR was associated with post-ketamine bradycardia but not with changes in BP. Baseline BP measurements were better determinants of post-ketamine changes in BP. Patients with lower baseline SBP, DBP, and MAP were significantly more likely to experience bradycardia (Figure 1) and hypotension (Figure 5) after ketamine administration. In contrast, patients with higher baseline SBP, DBP, and MAP were significantly more likely to develop hypertension after ketamine administration (Figure 8). Lower baseline SI was associated with post-ketamine hypotension (Figure 5), while higher baseline SI was associated with bradycardia (Figure 1) and hypertension (Figure 8). Age was an important determinant of post-ketamine response. Patients who experienced bradycardia (Figure 2) and hypotension (Figure 6) were on average 10-13 years older and those who experienced hypertension were on average 4 years younger (Figure 9) than those who did not experience either. Medical patients were more likely to experience bradycardia (Figure 3) and hypotension (Figure 7) after ketamine administration, while trauma patients were more likely to experience hypertension (Figure 10). Higher doses of ketamine caused more bradycardia and hypertension than lower doses (Table 4). Particularly, extremely high doses $(>200$ $\mathrm{mg}$ ) were associated with bradycardia and moderately high doses (151-200 mg) were associated with hypertension. Post-ketamine hypotension did not seem to be related to ketamine dose on the bivariate analysis.

On multivariate regression analysis, older patients were slightly more likely to experience bradycardia and hypotension after ketamine administration than younger patients. Also, trauma patients were 1.67 times more likely to develop hypertension than medical patients. Interestingly, the multivariate logistic regression showed baseline composite hemodynamic measures such as MAP and SI to be significant predictors of the ketamine response but not the non-composite measures such as HR, SBP, and DBP. Higher baseline MAP was found to slightly increase the risk of hypertension, but it was somewhat protective against bradycardia and hypotension. Baseline SI was found to be a positive predictor of post-ketamine bradycardia with the odds of experiencing bradycardia after ketamine administration increasing by over two-folds for every unit increase in baseline SI. Also, patients who received ketamine doses $>150 \mathrm{mg}$ were 1.3 times more likely to develop hypotension or hypertension and 1.77 times more likely to experience bradycardia after ketamine administration when compared to patients who received doses $<150 \mathrm{mg}$. Lastly, we tested the ketamine dose as a moderator of potential predictors of ketamine outcomes and found that ketamine dose only affects the relationship between pre-ketamine SI and post-ketamine bradycardia. The increase in the - odds of bradycardia for changes in SI was greater at lower doses of ketamine and less dependent on baseline SI values at higher doses (Figure 4). This may partially be explained by the increased occurrence of hypertension at high ketamine doses, which may have caused a reflex bradycardia that may not otherwise have happened in apparently lower risk patients (i.e. those with lower SI). Nonetheless, it is also possible that at higher doses $(>200 \mathrm{mg}$ ) ketamine may directly affect the heart in both low and high SI patients. It seems that ketamine dose is an important determinant of hemodynamic changes that follow its administration. The concept of a continuum of sedation and dose-dependent adverse events as a characteristic of non-dissociative sedatives is familiar to physicians [23]. Evidence suggests that ketamine does not exhibit any such dose-related adverse events within the range of clinically administered doses using standard administration techniques [24], [25]. However, most of the evidence comes from published studies that looked at side effects other than cardiovascular changes. We believe that ours was the first study to document dose as a moderator of the hemodynamic response to ketamine. In particular, ketamine appears to affect bradycardia and hypertension, in a dose-dependent fashion, and the dose of ketamine can moderate how other factors affect the post-ketamine outcome.

When comparing our study to previous studies that looked at similar cardiovascular parameters in the context of ketamine use in either a pre-hospital or emergency setting, we found similar results with some 
differences that may be explained by various factors such as sample size, and differing definitions of endpoints. Price et al. [26] conducted a retrospective study that compared the hemodynamic effects of etomidate with that of ketamine in 100 patients undergoing ETI in a pre-hospital setting. The researchers assessed up to two sets of VS before ETI and up to five sets of VS after ETI. The median pre-ketamine HR was 99-100 bpm and the median post-ketamine HR was 92-101 bpm. The median pre-drug SBP was 128-140 mmHg and the median post-drug SBP was 123-137 mmHg. Similar to their study, in our study, the mean pre-ketamine was HR 100.8 and SBP was $126.3 \mathrm{mmHg}$ while the mean post-ketamine HR was 101.6 and SBP was $122 \mathrm{mmHg}$ (Table 1). The rates of occurrence of bradycardia (12\% vs. 9.3\%), hypotension (24\% vs. 36,1\%), and hypertension (30\% vs. $51 \%$ ) in their study and our study respectively were also comparable. We observed slightly greater percentages of hemodynamic events than Price et al. [26] and this may be due to differences in the way the events were defined and the fact that we did not specifically count those who developed new cardiovascular abnormalities but rather counted the total number of post-ketamine endpoints. Sibley et al. [27] prospectively reviewed the use of ketamine to facilitate ETI in 71 patients in an HEMS setting and found ketamine to be an effective agent with similar complication rates to the control agent. In their study, they reported bradycardia in one (1\%), hypotension in five $(7 \%)$, and hypertension in four $(6 \%)$ patients [27]. The rates of hemodynamic events in their study were significantly lower than in our study. Also, the changes in MAP $(2.3 \mathrm{mmHg})$ and $\mathrm{HR}(0.45 \mathrm{bpm})$ after ketamine administration did not reach statistical significance in their study [27]. Their inability to detect any difference between pre- and post-ketamine MAP could be due to reduced power. Our study detected significant differences between pre- and post- ketamine MAP (Table 3) but not HR. Ballow et al. [28] compared the outcomes in 177 patients who received a ketamine protocol for RSI to 266 patients who received pre-protocol regimens that included midazolam, fentanyl, and etomidate with a muscle relaxant and reported hypotension in only $3.6 \%$ of patients in the ketamine group compared to $6 \%$ in the nonketamine group. Their study, despite the reasonable sample size, reported significantly lower hypotensive events than our study. It appears that the development of hemodynamic complications after ketamine administration probably depends on many factors that include, but are not limited to patient factors, and perhaps ketamine doses.

Studies that have looked at the role of baseline SI in predicting the development of hemodynamic events following ketamine for RSI in trauma and emergency patients are very scarce. Among the published literature, only one study described how baseline SI relates to hemodynamic changes after ketamine induction in the out-hospital setting [29] . In this study, researchers found that patients with a high SI exhibited blunted hypertensive responses and more frequent hypotension following ketamine induction, whereas patients with a low SI had sustained increases in HR and SBP [29]. These results agree with our findings. Patients who were likely to develop shock were more likely to develop hypotension after ketamine. On the other hand, patients with lower SI scores were less likely to develop hypotension but were more likely to develop hypertension and bradycardia. It is well known that a high SI predicts mortality in acute polytrauma patients [30], [31]. A more recent study showed that elevated SI predicts death in all trauma patients, but low SI values predicted death in head injured patients. Unfortunately, we did not test SI as an outcome to determine which factors determined higher or lower SI, but we did find a significant drop in the SI from $1.35 \pm .55$ at baseline to $1.28 \pm .53$ after ketamine on pre-post ketamine vs. testing.

In summary, we can clearly state that despite ketamine's cardiovascular "boosting "effect that renders it highly desirable in trauma patients who are frequently hypotensive or in shock, it may cause hemodynamic instability in select patients. Ketamine increases the central nervous system sympathetic outflow and decreases the reuptake of catecholamines producing an increase in BP, HR and stroke volume while maintaining systemic vascular resistance. On the other hand, it can also cause cardiac depression after induction. Ketamine's cardiac depressant effects are commonly overlooked because these effects are masked by its ability to increase sympathetic outflow. Therefore, in patients who are not catecholamine depleted, ketamine will produce a dose-related rise in the heart rate-systolic blood pressure product, leading to a transient rise in the cardiac index [32], [33] while in patients who are apparently catecholamine depleted 
Sheth et al., Adv.J. Grad. Res.; Vol. 3 Issue 1, pp: 20-33, January 2018

and lack autonomic control will experience direct myocardial depressant effects, leading to dose-dependent negative inotropic and chronotropic effects [34], [35]. This may explain why we observed hypotension and bradycardia more frequently in medical patients who probably are severely ill and catecholamine depleted given their need for intubation. It may also indirectly explain why hypotension was less associated with ketamine dose, as physicians are more likely to give lower doses of ketamine to the more severely ill patients with multiple comorbidities, who are the same patients that are more likely to develop hypotension with ketamine. Nonetheless, ketamine remains a useful anesthetic for RSI and in many studies has proven to be as effective as the gold standard "etomidate" with some favoring ketamine in some circumstances [19] .

\section{Conclusion}

In conclusion, baseline vital signs (HR, SBP, DBP, MAP, and SI) may help predict the hemodynamic response to ketamine during RSI. The results of this study indicate that older age, lower baseline vitals, and being a medical patient versus a trauma patient is more likely to predict the development of bradycardia and/or hypotension following the administration of ketamine during RSI. On the other hand, younger subjects, higher baseline vitals and trauma patients were more likely to develop hypertension following ketamine administration. Furthermore, the results suggest that higher doses of ketamine were associated with developing bradycardia and hypotension or hypertension. Understanding how baseline vital signs effect the response to ketamine administration will help first responders and trauma/emergency personnel better identify who may benefit from ketamine versus those who may potentially develop adverse events such as hypotension and bradycardia. This will enable healthcare professionals to improve upon current mortality and morbidity rates associated with RSI. Our study also provides key information to help researchers better design future studies to develop succinct guidelines for the use of ketamine in RSI.

\section{Acknowledgements}

The authors would like to especially thank PHI Air Medical Group for providing the data and for their support throughout the duration of the project. In addition, a special thank you to both Dr. Matthew Siderhurst and Dr. Daniel Showalter for their help and expertise in statistical analysis.

\section{How to Cite this Article:}

M. Sheth, A. Brand, and J. Halterman, "Ketamine-induced Changes in Blood Pressure and Heart Rate in Pre-hospital Intubated Patients", Advanced Journal of Graduate Research, vol. 3, no. 1, pp. 20-33, Oct. 2017. doi: https://doi.org/10.21467/ajgr.3.1.20-33

\section{References}

[1] AR Nakstad, HJ Heimdal, T Strand, M Sandberg."Incidence of desaturation during prehospital rapid sequence intubation in a physician-based helicopter emergency service," Am J Emerg Med, vol. 29, no.6, p. 639-44, 2011.

[2] SA Bernard, V Nguyen, P Cameron, K Masci, M Fitzgerald, DJ Cooper, T Walker, Std BP, P Myles, L Murray et al. "Prehospital rapid sequence intubation improves functional outcome for patients with severe traumatic brain injury: a randomized controlled trial," Ann Surg, vol. 252, no. 6, p. 959-65, 2010.

[3] A Timmermann, C Eich, SG Russo, U Natge, A Brauer, WH Rosenblatt, U Braun. "Prehospital airway management: a prospective evaluation of anaesthesia trained emergency physicians," Resuscitation, vol. 70, no.2, p. 179-85, 2006.

[4] M Eckstein, L Chan, A Schneir, R Palmer. "Effect of prehospital advanced life support on outcomes of major trauma patients," $J$ Trauma, vol. 48, no.4, p.643-8, 2000.

[5] B Burns, K Habig, H Eason, S Ware. "Difficult Intubation Factors in Prehospital Rapid Sequence Intubation by an Australian Helicopter Emergency Medical Service," Air Medical Journal, vol. 35, no.1, p.28-32, 2015.

[6] MT Cudnik, CD Newgard, M Daya, J Jui. "The impact of rapid sequence intubation on trauma patient mortality in attempted prehospital intubation," J Emerg Med, vol. 38, no.2, p. 175-81, 2010.

[7] ZB Perkins, M Gunning, J Crilly, D Lockey, B O'Brien. "The haemodynamic response to pre-hospital RSI in injured patients," Injury, vol. 44, no.5, p. 618-23, 2013.

[8] HE Wang, DP Davis, MA Wayne, T Delbridge. "Prehospital rapid-sequence intubation--what does the evidence show? Proceedings from the 2004 National Association of EMS Physicians annual meeting," Prehosp Emerg Care, vol. 8, no.4, p. 366-77, 2004.

[9] RS Sehdev, DA Symmons, K Kindl. "Ketamine for rapid sequence induction in patients with head injury in the emergency department," Emerg Med Australas, vol. 18, no.1, p.37-44, 2006.

[10] SJ Mercer. "The drug of war-a historical review of the use of ketamine in military conflicts," J Royal Naval Medical Serv., vol. 95 , no.3, p. 145-150, 2009.

[11] JD Tobias, M Leder. "Procedural sedation: A review of sedative agents, monitoring, and management of complications," Saudi $J$ Anaesth, vol. 5, no.4, p. 395-410, 2011. 
[12] K Porter. "Ketamine in prehospital care," Emerg Med J, vol. 21, no.3, p. 351-4, 2004.

[13] D Scherzer, M Leder, JD Tobias. "Pro-con debate: etomidate or ketamine for rapid sequence intubation in pediatric patients," J Pediatr Pharmacol Ther, vol. 17, no.2, p.142-9, 2012.

[14] V Ayatollahi, S Behdad, S Kargar, T Yavari. "Comparison of effects of ephedrine, lidocaine and ketamine with placebo on injection pain, hypotension and bradycardia due to propofol injection: a randomized placebo controlled clinical trial," Acta Med Iran, vol. 50, no. 9, p. 609-14, 2012.

[15] S Rajan, A Hassain, N Puthenveettil, L Kumar. "Efficacy and safety of low-dose ketamine as an adjunct analgesic and amnesic during caesarean section under general anaesthesia," Indian J Anaesth, vol. 59, no.10, p. 653-7, 2015.

[16] FK Özkan, Ziya; Süren, Mustafa. "The effect of intravenous ketamine on prevention of hypotension during spinal anesthesia in patients with benign prostatic hyperplasia," Nobel Medicus Journal, vol. 7, no. 3, p.82, 2011.

[17] JY Kim, JS Lee, HY Park, YB Kim, Y Kwon, HJ Kwak. "The effect of alfentanil versus ketamine on the intubation condition and hemodynamics with low-dose rocuronium in children," J Anesth, vol. 27, no.1, p. 7-11, 2013.

[18] J Barois, P Tourneux. "Ketamine and atropine decrease pain for preterm newborn tracheal intubation in the delivery room: an observational pilot study," Acta Paediatr, vol. 102, no. 12, p. e534-8, 2013.

[19] R Abbasivash, MM Aghdashi, B Sinaei, F Kheradmand. "The effects of propofol-midazolam-ketamine co-induction on hemodynamic changes and catecholamine response," J Clin Anesth, vol. 26, no. 8, p. 628-33, 2014.

[20] OK Losvik, MK Murad, E Skjerve, H Husum. "Ketamine for prehospital trauma analgesia in a low-resource rural trauma system: a retrospective comparative study of ketamine and opioid analgesia in a ten-year cohort in Iraq. Scand," J Trauma Resusc Emerg Med, vol. 23, p.94, 2015.

[21] KP Tran, Q Nguyen, XN Truong, V Le, VP Le, N Mai, H Husum, OK Losvik. "A comparison of ketamine and morphine analgesia in prehospital trauma care: a cluster randomized clinical trial in rural Quang Tri province, Vietnam.," Prehosp Emerg Care, vol. 18, no.2, p. 257-64, 2014.

[22] MY Rady, HA Smithline, H Blake, R Nowak, E Rivers. "A comparison of the shock index and conventional vital signs to identify acute, critical illness in the emergency department," Ann Emerg Med, vol. 24, no.4, p. 685-90, 1994.

[23] SM Green, MG Roback, RM Kennedy, B Krauss."Clinical practice guideline for emergency department ketamine dissociative sedation: 2011 update," Ann Emerg Med, vol. 57, no.5, p. 449-61, 2011.

[24] SM Green, N Kuppermann, SG Rothrock, CB Hummel, M Ho. "Predictors of adverse events with intramuscular ketamine sedation in children," Ann Emerg Med, vol. 35, no.1, p.35-42, 2000.

[25] SM Green, MG Roback, B Krauss, L Brown, RG McGlone, D Agrawal, M McKee, M Weiss, RD Pitetti, MA Hostetler et al. "Predictors of Airway and Respiratory Adverse Events With Ketamine Sedation in the Emergency Department: An Individual-Patient Data Meta-analysis of 8,282 Children," Annals of Emergency Medicine, vol.5, no. 2, p. 158-168, e4, 2009.

[26] B Price, AO Arthur, M Brunko, P Frantz, JO Dickson, T Judge, SH Thomas. "Hemodynamic consequences of ketamine vs etomidate for endotracheal intubation in the air medical setting," Am J Emerg Med, vol. 31, no.7, p. 1124-32, 2013.

[27] A Sibley, M Mackenzie, J Bawden, D Anstett, C Villa-Roel, BH Rowe. "A prospective review of the use of ketamine to facilitate endotracheal intubation in the helicopter emergency medical services (HEMS) setting," Emerg Med J, vol. 28, no.6, p. 521-5, 2011.

[28] SL Ballow, KL Kaups, S Anderson, M Chang. "A standardized rapid sequence intubation protocol facilitates airway management in critically injured patients," Journal of Trauma and Acute Care Surgery, vol. 73, no.6, p. 1401-1405, 2012.

[29] M Miller, N Kruit, C Heldreich, S Ware, K Habig, C Reid, B Burns. "Hemodynamic Response After Rapid Sequence Induction With Ketamine in Out-of-Hospital Patients at Risk of Shock as Defined by the Shock Index," Ann Emerg Med, vol. 68, no.2, p.181-188 e2, 2016.

[30] KF Montoya, JD Charry, JS Calle-Toro, LR Núñez, G Poveda."Shock index as a mortality predictor in patients with acute polytrauma," Journal of Acute Disease, vol. 4, no. 3, p. 202-204, 2015.

[31] V Pandit, P Rhee, A Hashmi, N Kulvatunyou, A Tang, M Khalil, T O'Keeffe, D Green, RS Friese, B Joseph. "Shock index predicts mortality in geriatric trauma patients: an analysis of the National Trauma Data Bank," J Trauma Acute Care Surg, vol. 76, no.4, p. $1111-5,2014$

[32] WA Tweed, M Minuck, D Mymin. "Circulatory responses to ketamine anesthesia," Anesthesiology, vol. 37, no.6, p. 613-9, 1972.

[33] PF White, WL Way, AJ Trevor. "Ketamine--its pharmacology and therapeutic uses," Anesthesiology, vol. 56, no.2, p. 119-36, 1982.

[34] DA Schwartz, LD Horwitz. "Effects of ketamine on left ventricular performance," Journal of Pharmacology and Experimental Therapeutics, vol. 194, no.2, p. 410-414, 1975.

[35] DL Traber, RD Wilson RD, LL Priano. "Differentiation of the Cardiovascular Effects of CI-581," Anesthesia \& Analgesia, vol. 47, no.6, p. 769-778, 1968.

\section{Publish your research article in AIJR journals-}

$\checkmark$ Online Submission and Tracking

$\checkmark$ Peer Reviewed

$\checkmark$ Rapid decision

$\checkmark$ Immediate Publication after acceptance

$\checkmark$ Open Access (Articles freely available online)

$\checkmark$ Retain full copyright of your article.

Submit your article at journals.aijr.in 